\title{
Differences in Discriminability and Response Bias on Rey Auditory Verbal Learning Test Delayed Recognition in Behavioral Variant Frontotemporal Dementia and Alzheimer's Disease
}

\author{
E. van den Berg ${ }^{1}$ (1) , J.M. Poos ${ }^{1}$, L.C. Jiskoot ${ }^{1,2}$, L.M. Heijnen ${ }^{1}$, S. Franzen ${ }^{1}$ (1) , R.M.E. Steketee ${ }^{3}$, R. Meijboom ${ }^{3,4,5}$, \\ F.J. de Jong ${ }^{1}$, H. Seelaar ${ }^{1}$, J.C. van Swieten ${ }^{1}$ and J.M. Papma ${ }^{1}$ (1) \\ ${ }^{1}$ Department of Neurology, Erasmus MC University Medical Center, Rotterdam, The Netherlands \\ ${ }^{2}$ Dementia Research Center, University College London, London, UK \\ ${ }^{3}$ Department of Radiology and Nuclear Medicine, Erasmus MC University Medical Center, Rotterdam, The Netherlands \\ ${ }^{4}$ Edinburgh Imaging and Centre for Clinical Brain Sciences, University of Edinburgh, Edinburgh, UK \\ ${ }^{5}$ UK Dementia Research Institute Edinburgh, University of Edinburgh, Edinburgh, UK
}

(Received August 19, 2019; Final Revision March 9, 2020; AcCePted March 18, 2020; First Published Online April 27, 2020)

\begin{abstract}
Objective: Episodic memory is impaired in Alzheimer's disease (AD) dementia but thought to be relatively spared in behavioral variant frontotemporal dementia (bvFTD). This view is challenged by evidence of memory impairment in bvFTD. This study investigated differences in recognition memory performance between bvFTD and AD.

Method: We performed a retrospective analysis on the recognition trial of the Rey Auditory Verbal Learning Test in patients with bvFTD $(n=85), \mathrm{AD}(n=55)$, and control participants $(n=59)$. Age- and education-adjusted betweengroup analysis was performed on the total score and indices of discriminative ability and response bias. Correlations between recognition and measures of memory, language, executive functioning, and construction were examined. Results: Patients with AD had a significantly lower total recognition score than patients with bvFTD (control $28.8 \pm 1.5$; bvFTD $24.8 \pm 4.5$; AD 23.4 $\pm 3.6, p<.01$ ). Both bvFTD and AD had worse discriminative ability than controls (A' control $0.96 \pm 0.03$; bvFTD $0.87 \pm 0.03$; AD $0.84 \pm 0.10, p<.01$ ), but there was no difference in response bias (B" control $0.9 \pm 0.2$; bvFTD $1.6 \pm 1.47$; AD 1.4 $\pm 1.4, p<.01$ ). AD had worse discriminability than bvFTD $(p<.05)$. Discriminability was associated with memory for both patient groups (median correlation coefficient $r=.34$ ) and additionally associated with language $(r=.31)$, but not executive functioning $(r=-.03)$ in bvFTD. Response bias was unrelated to other cognitive functions $(r=-.02)$. Conclusions: Discriminability, but not response bias, differentiated patients with bvFTD from AD. The presence of an impaired discrimination index suggests a "pure" (recognition) memory deficit in bvFTD.
\end{abstract}

Keywords: Memory, bvFTD, Cognition, Executive functioning, Language, Diagnosis

\section{INTRODUCTION}

Behavioral variant frontotemporal dementia (bvFTD) and Alzheimer's disease (AD) are the two most common early onset dementias (Ratvanali, Brayne, Dawson, \& Hodges, 2002). In the clinical diagnostic phase, neuropsychological assessment is used to detect impairments in cognitive functioning and to differentiate between these two types of dementia. The cognitive profile of bvFTD is characterized by impaired executive functioning, social cognition, and language [related to atrophy in frontal and temporal brain

Correspondence and reprint requests to: E. van den Berg, Department of Neurology NF-331, PO Box 2040, 3000CA Rotterdam, The Netherlands. E-mail: e.vandenberg@erasmusmc.nl areas (Rohrer \& Rosen, 2013)], with relatively spared memory and construction (Rascovsky et al., 2011). Theoretically, this cognitive profile is markedly different from the profile of $\mathrm{AD}$, which is most commonly characterized by memory deficits resulting from atrophy of the medial temporal lobe (MTL; McKhann et al., 2011). In the last decade, this traditional view on the difference in cognitive profiles between bvFTD and AD has been challenged by multiple reports of (sometimes profound) memory impairments in patients with bvFTD, even in early disease stages (Glosser, Gallo, Clark, \& Grossman, 2002; Hornberger, Piguet, Graham, Nestor, \& Hodges, 2010; Kramer et al., 2005; Pennington, Hodges, \& Hornberger, 2011; Ricci, Graef, Blundo, \& Miller, 2012). Likewise, patients with AD may present 
with executive dysfunctioning and/or significant "frontal" behavioral symptoms (Ossenkoppele et al., 2015), making the differentiation between bvFTD and AD in clinical practice particularly challenging.

Systematic investigations of episodic memory functioning in bvFTD are increasingly reported but show inconsistent results (for review, see Poos, Jiskoot, Papma, van Swieten, \& van den Berg, 2018) that are only partly explained by differences in testing procedures (verbal $v s$. visual memory tests; free $v s$. cued recall) and variation in patient samples (e.g., inclusion of nonprogressive "phenocopy" bvFTD patients, disease duration, and heterogeneous clinical presentation). Several studies report memory deficits in bvFTD (compared with controls) that are equal in nature and extent to those found in AD (e.g., Gregory, Orrell, Sahakian, \& Hodges, 1997; Hornberger et al., 2010; Pennington et al., 2011; Ricci et al., 2012). Others, however, demonstrate relative sparing of memory performance in bvFTD compared with AD (e.g., Thompson, Stopford, Snowden, \& Neary, 2005). Free delayed recall measures appear to best discriminate AD from bvFTD, with patients with AD performing worse than bvFTD (Heidler-Gary \& Hillis, 2007; Ricci et al., 2012), although this difference is not found invariably (Hornberger et al., 2010; Pennington et al., 2011).

Episodic memory impairment in bvFTD is commonly viewed as a consequence of executive dysfunctioning (i.e., poor organization, lack of efficient retrieval strategies; Hornberger et al., 2010; Kramer et al., 2005) rather than "pure" or primary amnesia. In contrast, Glosser et al. (2002) showed that recall performance in bvFTD is not enhanced by cueing (use of semantic clusters), indicating that memory impairments in bvFTD are associated with primary encoding deficits rather than suboptimal retrieval strategies. In addition, recent studies show impaired memory performance in patients with bvFTD even after controlling for executive load (Bertoux et al., 2014; Lemos, Duro, Simões, \& Santana, 2014). These findings are not surprising as several brain structures important for memory performance, such as the MTL (including the hippocampus and supporting structures) and brain regions that connect the MTL to prefrontal areas (such as the fornix) (Rugg, Otten, \& Henson, 2002) are implicated not only in AD but in bvFTD as well (Hornberger \& Piguet, 2012). Indeed, deficits in delayed recall in both AD and FTD rely not only on the MTL but on the integrity of prefrontal areas as well (Frisch et al., 2013; Irish, Piguet, Hodges, \& Hornberger, 2014; Wong, Flanagan, Savage, Hodges, \& Hornberger, 2014).

The majority of studies investigating episodic memory performance in bvFTD and AD focus on measures of immediate or delayed (free) recall, most commonly with verbal tests such as the Rey Auditory Verbal Learning Test (RAVLT). Recognition memory is also routinely assessed as part of these memory tests (both in clinical practice and in scientific research) but has received much less attention. This is unfortunate as recognition memory paradigms may provide crucial information on memory and executive processes aiding the differentiation between
AD and bvFTD. Recognition memory entails patients to indicate whether a certain stimulus was previously encountered ("old") or new. Patients with bvFTD tend to outperform AD patients in recognition memory (Hornberger et al., 2010) and sometimes even show no impairment compared to control participants (Pasquier, Grymonprez, Lebert, Van der Linden, 2001; Ricci et al., 2012). This finding may be attributed to the fact that cueing in recognition memory tasks enables patients with bvFTD to overcome retrieval problems (at least to some extent), but the recognition deficits in $\mathrm{AD}$ reflect true forgetting of the items. Under certain conditions, patients with bvFTD may thus exhibit a greater ability to discriminate "old" from "new" items than patients with AD. Interestingly, differences in performance on recognition memory tasks between bvFTD and AD are not found invariably (see, e.g., Glosser et al., 2002; Pennington et al., 2011), suggesting that certain characteristics of the recognition memory tasks (e.g., number of distractors, type of cueing) may elicit a trend or bias toward a more liberal (tendency to respond "yes" to any item that is presented) or conservative ("no" tendency) response. Theoretically, the ability to distinguish target words from distractor words ("discriminability") is indeed independent from the tendency to favor "yes" or "no" responses when there is uncertainty about the correct response ("response bias") (Snodgrass \& Corwin, 1988). A person can thus exhibit a liberal response bias when discriminative ability is either high or low (Kramer et al., 2005). Patients with AD generally have a more liberal response bias (Beth, Budson, Waring, \& Ally, 2009; Budson, Wolk, Chong, \& Waring, 2006), resulting in an increase in false positive responses ("yes" tendency) associated with both prefrontal and (para)hippocampal areas (Budson et al., 2006). Response bias in bvFTD has only been scarcely examined. Recent work by Flanagan et al. (2016) shows a higher rate of false positive responses in both $\mathrm{AD}$ and bvFTD that was most strongly correlated with measures of disinhibition in the latter. As of yet, it remains unclear whether discriminability and response bias as such are valuable measures in discriminating $\mathrm{AD}$ from bvFTD.

The aims of the present study were (1) to examine differences in recognition memory performance on a widely used verbal memory test (RAVLT) between patients with bvFTD and AD, (2) to specifically compare measures of discrimination and response bias between these groups, and (3) to investigate associations between recognition memory and other measures of memory, language, executive functioning within and between the groups, and disease severity.

\section{METHOD}

\section{Participants}

Retrospective data from 140 patients ( 85 bvFTD, $55 \mathrm{AD}$ ) and 59 control participants were included. Patients visited the memory clinic of the Erasmus MC University Medical Center between 2005 and 2018 for a standardized workup consisting of a neurological and neuropsychological 
assessment, laboratory testing (including lumbar puncture in subsample), and brain imaging. Clinical diagnoses were made in a multidisciplinary consensus meeting with an experienced neurologist, geriatrician, neuropsychologist, and radiologist. All patients with bvFTD met core clinical diagnostic criteria for bvFTD with insidious onset, decline in social behavior and personal conduct, emotional blunting, and loss of insight reported by caregivers (Neary et al., 1998; Rascovsky et al., 2011). Memory complaints and impaired episodic memory performance were allowed for if the other core diagnostic criteria were present. Nonprogressive (i.e., phenocopy bvFTD) patients were excluded as these patients present with little or no memory dysfunction (Pennington et al., 2011). Thirty-four patients were part of an ongoing epidemiological study of Dutch pathologically confirmed genetic FTD families [Dopper et al., 2014; progranulin $(G R N) n=12$; microtubule-associated protein tau (MAPT) $n=9$; chromosome 9 open reading frame 72 (C9orf72) $n=13$ ]. Patients with AD met the NINCDS-ADRDA criteria for probable AD (McKhann et al., 2011). Control participants were included from two previous studies $(n=28$, Papma et al., 2014; $n=31$, Steketee et al., 2016). These control participants reported no history of neurological (e.g., major stroke, brain tumor, and epilepsy) or severe psychiatric disorder (e.g., major depression, substance abuse) negatively affecting cognition and a normal brain MRI. The study was approved by the local medical ethics committee. The research was completed in accordance with the Helsinki Declaration. All participants gave written informed consent.

\section{Neuropsychological Assessment}

Neuropsychological assessment was performed as part of the memory clinic work-up. Although the neuropsychological test battery was standardized, adaptations were made for individual patients according to type of symptoms and severity. Neuropsychological tests that were performed by $<50 \%$ of participants were excluded from the analysis. Global cognitive functioning was screened by means of the Mini-Mental State Examination (MMSE; Folstein, Folstein, \& McHugh, 1975) and the Frontal Assessment Battery (FAB; Dubois, Slachevsky, Litvan, \& Pillon, 2000).

Verbal learning and memory were assessed using the Dutch version of the RAVLT (van der Elst, van Boxtel, van Breukelen, \& Jolles, 2005). The RAVLT is a supraspan verbal learning test consisting of 5 learning trials of 15 words, followed by a $20-30$-min delayed free recall and a 30 -word delayed recognition trial including al 15 words from the learning trials and 15 unrelated words. For the purpose of the present analysis, the following parameters were derived from the delayed recognition trial of the RAVLT:

1. Total score of delayed recognition (0-30)

2. Number of correctly recognized words in the recognition trial ("hits," 0-15)

3. Number of incorrectly recognized words in the recognition trial ("false positives," $0-15$ ).
Level of performance on a yes-no recognition memory test is reflected in the number of correct hits and false positive errors. These data yield two measures of recognition memory (adapted from signal detection theory): recognition discriminability (the ability to distinguish target words from distractor words) and response bias (the tendency to favor "yes" or "no" responses when there is uncertainty about the correct response) (Kramer et al., 2005). Calculation of hits minus false positives is a widely used and easily obtained measure of discriminability, and the ratio between "yes" and "no" answers entails a (crude) estimation of response bias. Snodgrass and Corwin provide a more elaborate distributionfree (nonparametric) model for calculating discriminability (A') and response bias (B") that is specifically suitable for a population of persons that may have poor discrimination performance (e.g., persons with dementia) and can be applied to non-normally distributed data (Snodgrass \& Corwin, 1988). Discrimination index A' and response bias B" are estimated by formulas (1) and (2):

$$
\begin{array}{r}
\mathrm{A}^{\prime}=0.5+((\text { hits }- \text { false alarm })(1+\text { hits }- \text { false alarms })) / \\
((4 * \text { hits }(1-\text { false alarms }))
\end{array}
$$

$$
\begin{aligned}
\mathrm{B}^{\prime \prime}= & (\text { hits }(1-\text { hits })-\text { false alarms }(1-\text { false alarms })) / \\
& (\text { hits } *(1-\text { hits })+\text { false alarms }(1-\text { false alarms }))
\end{aligned}
$$

We chose to investigate both the simple and the more elaborate measures of discriminability and response bias:

\footnotetext{
4. Discrimination index "hits minus false positives"

5. Discrimination index A'

6. Response bias yes/no ratio

7. Response bias index B".
}

Other neuropsychological tests that were included in the present analysis were the story recall subtest of the Rivermead Behavioral Memory Test (RBMT; immediate recall score 0-42, delayed recall \% retained; Wilson, Cockburn, \& Baddeley, 1985), the Visual Association Test (VAT; score 0-12; Lindeboom, Schmand, Tulner, Walstra, \& Jonker 2002), the Boston Naming Test (BNT; score 0-60; Kaplan, Goodglass, \& Weintraub, 1978), category fluency (animals, 1 min), letter fluency (letters D-A-T which are the Dutch equivalent of F-A-S (Schmand, Groenink, \& van den Dungen, 2008), modified Wisconsin Card Sorting Test (WCST; concepts 0-6; Nelson, 1976), Trailmaking Test A and B (TMT; time for card $\mathrm{A}$ and $\mathrm{B}, \mathrm{B} / \mathrm{A}$ ratio score; Corrigan \& Hinkeldey, 1987), Stroop Color-Word Test (time for card I, II, and III, card III/II interference ratio score; Stroop, 1935), and Clock drawing test (score 0-14; after Royall, Cordes, \& Polk, 1998). 
Table 1. Characteristics of the patients with bvFTD, AD, and the control group

\begin{tabular}{|c|c|c|c|c|}
\hline & bvFTD & $\mathrm{AD}$ & Control & $p$ Value for difference ${ }^{a}$ \\
\hline$n$ & 85 & 55 & 59 & \\
\hline Age & $62.0 \pm 9.0$ & $68.1 \pm 7.2$ & $67.0 \pm 6.8$ & $<.01(\mathrm{bvFTD}<\mathrm{AD}=\mathrm{con})$ \\
\hline Male sex $(\%)$ & $51(60)$ & $32(58)$ & $35(59)$ & ns \\
\hline Education $^{\mathrm{b}}$, median (IQR) & $5(4-6)$ & $5(4-6)$ & $5(5-6)$ & $<.01(\mathrm{bvFTD}=\mathrm{AD}<\mathrm{con})$ \\
\hline MMSE & $25.0 \pm 4.5$ & $22.7 \pm 4.6$ & $28.8 \pm 1.1$ & $<.01(\mathrm{AD}<\mathrm{bvFTD}<\mathrm{con})$ \\
\hline $\mathrm{FAB}^{\mathrm{c}}$ & $12.3 \pm 4.0$ & $12.6 \pm 4.1$ & - & ns \\
\hline Months since symptom onset & $30 \pm 26$ & $46 \pm 42$ & - & $.02(\mathrm{bvFTD}<\mathrm{AD})$ \\
\hline \multicolumn{5}{|l|}{ Cognitive tests } \\
\hline RAVLT total trial $1-5(0-75)$ & $28.3 \pm 11.5$ & $23.8 \pm 9.0$ & $44.6 \pm 8.7$ & $<.01(\mathrm{AD}=\mathrm{bvFTD}<\mathrm{con})$ \\
\hline RAVLT delayed recall $(0-15)$ & $4.8 \pm 3.8$ & $2.7 \pm 2.7$ & $9.3 \pm 2.9$ & $<.01(\mathrm{AD}<\mathrm{bvFTD}<\mathrm{con})$ \\
\hline RAVLT \% savings $(0-100)^{\mathrm{d}}$ & $59 \pm 36$ & $40 \pm 39$ & $82 \pm 17$ & $<.01(\mathrm{AD}<\mathrm{bvFTD}<\mathrm{con})$ \\
\hline RBMT story immediate recall $(0-42)$ & $11.4 \pm 10.3$ & $7.5 \pm 4.4$ & $18.7 \pm 5.6$ & $<.01(\mathrm{bvFTD}=\mathrm{AD}<\mathrm{con})$ \\
\hline RBMT story \% recall & $56.9 \pm 29.5$ & $46.6 \pm 34.7$ & $79.1 \pm 18.3$ & $<.01(\mathrm{bvFTD}=\mathrm{AD}<\mathrm{con})$ \\
\hline Visual Association Test (0-12) & $9.6 \pm 3.3$ & $5.6 \pm 4.0$ & $11.9 \pm 0.3$ & $<.01(\mathrm{AD}<\mathrm{bvFTD}<\mathrm{con})$ \\
\hline Boston Naming Test (0-60) & $40.3 \pm 12.4$ & $41.5 \pm 10.0$ & $55.6 \pm 3.2$ & $<.01(\mathrm{bvFTD}=\mathrm{AD}<\mathrm{con})$ \\
\hline Category fluency & $12.9 \pm 5.1$ & $12.2 \pm 5.2$ & $24.0 \pm 5.1$ & $<.01(\mathrm{bvFTD}=\mathrm{AD}<\mathrm{con})$ \\
\hline Letter fluency & $17.4 \pm 10.3$ & $19.7 \pm 10.8$ & $38.6 \pm 13.0$ & $<.01(\mathrm{bvFTD}=\mathrm{AD}<\mathrm{con})$ \\
\hline mWCST concepts $(0-6)$ & $2.6 \pm 1.8$ & $1.7 \pm 1.3$ & $5.5 \pm 1.1$ & $<.01(\mathrm{AD}<\mathrm{bvFTD}<\mathrm{con})$ \\
\hline Trailmaking A & $63.4 \pm 39.8$ & $94.6 \pm 55.8$ & $38.5 \pm 17.3$ & $<.01(\mathrm{AD}<\mathrm{bvFTD}<\mathrm{con})$ \\
\hline Trailmaking B & $191.8 \pm 92.4$ & $269.4 \pm 65.9$ & $83.5 \pm 34.6$ & $<.01(\mathrm{AD}<\mathrm{bvFTD}<\mathrm{con})$ \\
\hline Stroop Color-Word card III & $172.6 \pm 101.6$ & $227.3 \pm 124.4$ & $104.0 \pm 22.9$ & $<.01(\mathrm{AD}<\mathrm{bvFTD}<\mathrm{con})$ \\
\hline Clock drawing (0-14) & $10.5 \pm 2.2$ & $8.8 \pm 2.8$ & $12.6 \pm 1.1$ & $<.01(\mathrm{AD}<\mathrm{bvFTD}<\mathrm{con})$ \\
\hline
\end{tabular}

bvFTD, behavioral variant frontotemporal dementia; AD, Alzheimer's disease; con, control participants; IQR, interquartile range; MMSE, Mini-Mental State Examination; RAVLT, Rey Auditory Verbal Learning Test; RBMT, Rivermead Behavioral Memory Test; mWCST, modified Wisconsin Card Sorting Test.

a Between-group differences adjusted for age and level of education.

${ }^{\mathrm{b}}$ Level of education according to Verhage (1964) ( 1 = less than primary school, $7=$ university degree).

${ }^{\mathrm{c}}$ Frontal Assessment Battery, available in 27 patients with AD.

${ }^{\mathrm{d}}$ Defined as (delayed free recall/trial 5) $* 100$.

\section{Statistical analysis}

RAVLT recognition memory scores were compared between bvFTD, AD, and control participants with analysis of variance for normally distributed data or Kruskal-Wallis tests for nonparametric data, adjusted for age, education, and time since symptom onset. Post hoc pairwise comparisons (controls $v s$. patients groups, bvFTD vs. AD) were analyzed with Scheffe's tests and, in case of nonparametric data, with Mann-Whitney $U$ tests. Within the bvFTD group, differences in RAVLT recognition memory between sporadic versus genetic bvFTD and between patients with GRN, MAPT, and $C 9$ orf 72 mutations were also explored. Bivariate correlation was used to assess the relation between RAVLT recognition memory scores and other cognitive functions (Pearson's $r$ for normally distributed data or Spearman's $r$ for nonparametric data; adjusted for age and level of education). Statistical analyses were performed using SPSS Statistics 21.0 (IBM Corp., Armonk, NY, USA).

\section{RESULTS}

Table 1 shows the characteristics of the 199 participants. Patients with bvFTD were significantly younger than patients with $\mathrm{AD}$ and controls $\left[F(2,199)=12.3, p<.001, \eta^{2}=0.11\right]$. Both patient groups had a lower level of education compared with the control participants $[\mathrm{H}(2)=6.7, p<.05]$. As expected, patients with AD had the lowest MMSE score and the bvFTD group had an intermediate position between the $\mathrm{AD}$ and control group $\left[F(2,157)=19.4, p<.001, \eta^{2}=0.20\right]$. The $\mathrm{FAB}$ was administered to 67/86 patients with bvFTD and 27/55 patients with $\mathrm{AD}$ and showed no significant difference between groups $\left[F(1,94)=1.13, p=.79, \eta^{2}=0.001\right]$. Time since symptom onset (months) was shorter for bvFTD than $\mathrm{AD}[t(136)=-2.69, p<.01]$. As expected, both patient groups showed significantly poorer performance than control participants on all cognitive tests (Table 1). Compared with bvFTD, the patients with AD had lower scores on measures of memory (RAVLT, RBMT, and VAT), executive functioning (WCST, TMT, and Stroop), and construction (Clock drawing) (Table 1).

\section{Group Differences in RAVLT Recognition Memory}

With regard to performance on the RAVLT, patients with AD had a significantly lower delayed recall and percentage savings score than the bvFTD group $[F(1,140)=7.16, p<.01$, $\left.\eta^{2}=0.05\right]$, but there was no difference between the two patient groups in immediate recall $[F(1,140)=2.61, p=.11$, $\eta^{2}=0.02$ ) (see Table 1 and Figure 1). Table 2 shows the differences in RAVLT recognition scores. Patients with 


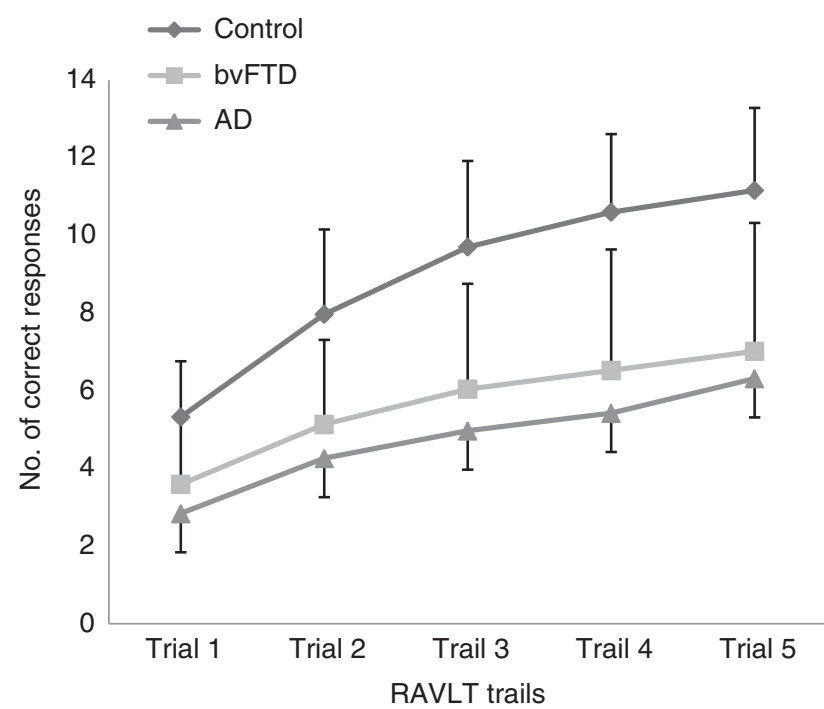

Fig. 1. Performance on the Rey Auditory Verbal Learning Test. bvFTD, behavioral variant frontotemporal dementia; AD, Alzheimer's disease.

bvFTD had a lower total recognition score and hit rate than the control participants and, in turn, the AD group performed significantly worse than the patients with bvFTD (total recognition score bvFTD vs. AD $U=1826, p<.05$; hits $U=1705, p<.01)$. Both patient groups had significantly more false positives than controls, but there was no difference in number of false positives between patients with AD and bvFTD (bvFTD vs. AD $U=2746, p=.08$ ). For the discrimination indices (hits-FA and A') both patients with $\mathrm{AD}$ and bvFTD had worse discriminative ability than controls $(H=62.8, p<.01)$ (Figure 2). Patients with AD had a lower A' than the bvFTD group, but there was no difference in "hits minus false positives" (discrimination index $\mathrm{A}^{\prime} U=1789, p<.05$; hits minus false positives $U=1846, p=.054$ ). An effect size calculation for the comparison of A' between patients with bvFTD and $\mathrm{AD}$ showed a medium effect size of 0.3-0.4, which corresponds to a 72.6-78.7\% overlap between the distributions (Zakzanis, 2001). With regard to the bias indices, both dementia groups showed a higher yes-no ratio and a lower B" than the controls (yes-no ratio $H=6.37$, $p<.05$; B" $H=9.02, p<.05$ ) representing a (slightly more) liberal response bias for the patients. There was no difference in response bias between patients with bvFTD and AD (yes-no ratio $U=2194, p=.54, d=0.12$ ). For both AD and bvFTD, the standardized total recognition score was lower than the standardized delayed recall score ( $z$-scores standardized on the control group; AD delayed recall $-2.3 \pm 0.9$, total recognition $-3.5 \pm 2.4$, $t(54)=4.51, p<.01$; bvFTD delayed recall $-1.6 \pm 1.3$, total recognition $-2.6 \pm 3.0, t(84)=4.34, p<.01$ ). There were no differences between patients with sporadic $(n=51) \quad$ versus genetic $(n=34)$ bvFTD (see Supplementary Table). In the subgroup of patient with a known genetic mutation for bvFTD $(n=34)$ no differences

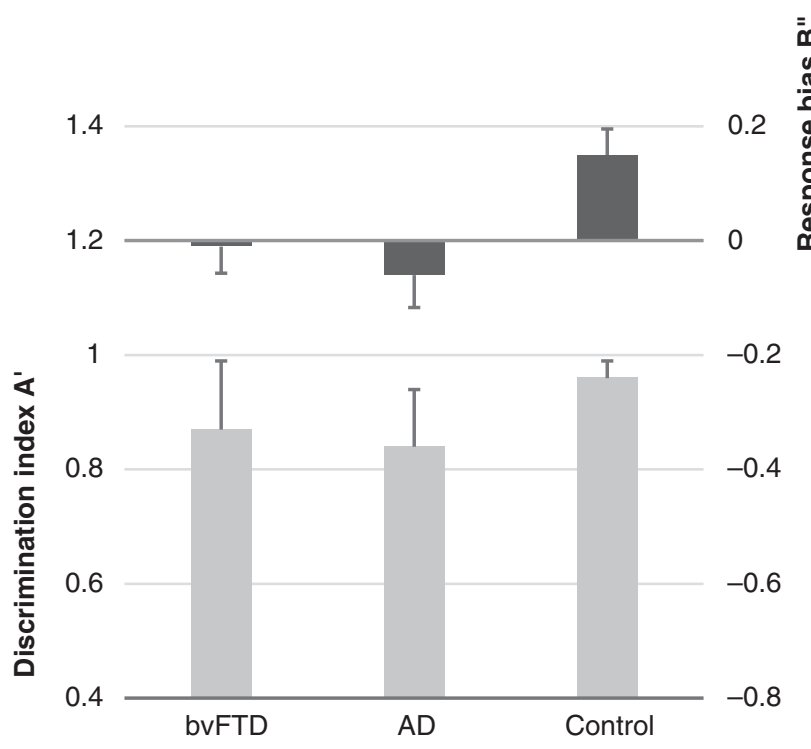

Fig. 2. Discrimination and response bias indexes. bvFTD, behavioral variant frontotemporal dementia; $\mathrm{AD}$, Alzheimer's disease.

were observed in $\mathrm{A}^{\prime}$ or B' (or any other measure of RAVLT) between GRN and MAPT mutation carriers and C9orf72 repeat expansion carriers (see Supplementary Table). Repeating the analysis in those patients with $\leq 24$ months of time since symptom onset versus $>24$ months yielded highly similar results (data not shown).

\section{Correlations with Other Cognitive Functions and Disease Severity}

The results of the correlation analysis (Table 3) showed that the discrimination indices (hits minus false positive and A') are associated with memory performance (RBMT, VAT) in the $\mathrm{AD}$ and bvFTD groups. In the bvFTD group, additional associations were found for measures of language (BNT, semantic, and letter fluency). There were no significant associations between the discrimination indices and measures of executive functioning or construction in either patient group. Similarly, there were no significant associations between indices of response bias (yes/no ratio and B") and any of the other cognitive functions. Correlation analysis in the control group showed no clear patterns of associations, most likely resulting from ceiling effects on the RAVLT recognition trial. With regard to disease severity, the correlation analysis showed that "time since symptom onset" was significantly correlated with delayed recognition $(r=-.23)$ and total hits $(r=-.17)$ but not with A' $(r=-.16)$ or B" $(.06)$. Age was also not significantly associated with the recognition variables (range $r-.04$ to -.15 ).

\section{DISCUSSION}

In the present study, we investigated differences recognition memory between patients with bvFTD and AD. The main results showed that both patients with bvFTD and AD had 
Table 2. Between-group differences in RAVLT recognition memory

\begin{tabular}{|c|c|c|c|c|c|}
\hline & bvFTD & $\mathrm{AD}$ & Control & Statistics $^{\mathrm{a}}$ & Group differences \\
\hline Delayed recognition (0-30) & $24.8 \pm 4.5$ & $23.4 \pm 3.6$ & $28.8 \pm 1.5$ & $H=62.9, p<.01$ & $\mathrm{AD}<\mathrm{bvFTD}<\mathrm{con}$ \\
\hline Total hits on recognition & $13.2 \pm 2.1$ & $12.1 \pm 2.6$ & $14.1 \pm 1.3$ & $H=28.8, p<.01$ & $\mathrm{AD}<\mathrm{bvFTD}<\mathrm{con}$ \\
\hline False positives on recognition & $3.4 \pm 4.3$ & $3.6 \pm 3.0$ & $0.3 \pm 0.6$ & $H=56.2, p<.01$ & $\mathrm{bvFTD}=\mathrm{AD}<\mathrm{con}$ \\
\hline Hits minus false positives & $9.7 \pm 4.5$ & $8.4 \pm 3.6$ & $13.8 \pm 1.5$ & $H=63.3, p<.01$ & $\mathrm{bvFTD}=\mathrm{AD}<\mathrm{con}$ \\
\hline Discrimination index $A^{\prime}$ & $0.87 \pm 0.12$ & $0.84 \pm 0.10$ & $0.96 \pm 0.03$ & $H=64.2, p<.01$ & $\mathrm{AD}<\mathrm{bvFTD}<\mathrm{con}$ \\
\hline Yes/no ratio & $1.6 \pm 1.7$ & $1.4 \pm 1.4$ & $0.9 \pm 0.2$ & $H=6.4, p<.05$ & $\mathrm{bvFTD}=\mathrm{AD}<\mathrm{con}$ \\
\hline Bias index B" & $-0.01 \pm 0.43$ & $-0.06 \pm 0.42$ & $0.15 \pm 0.35$ & $H=9.0, p<.05$ & $\mathrm{bvFTD}=\mathrm{AD}<\mathrm{con}$ \\
\hline
\end{tabular}

RAVLT, Rey Auditory Verbal Learning Test; bvFTD, behavioral variant frontotemporal dementia; AD, Alzheimer's disease; con, control participants.

${ }^{a}$ Analyses adjusted for age and level of education.

Table 3. Association between recognition memory indices and other cognitive functions in patients with bvFTD and AD

\begin{tabular}{|c|c|c|c|c|c|c|c|c|}
\hline & \multicolumn{4}{|c|}{ bvFTD } & \multicolumn{4}{|c|}{$\mathrm{AD}$} \\
\hline & Hits - false positives & $A^{\prime}$ & Yes/no ratio & B” & Hits - false positives & $A^{\prime}$ & Yes/no ratio & B” \\
\hline RBMT story immediate & $0.34 *$ & $0.31 *$ & -0.11 & -0.05 & $0.40 *$ & $0.36^{+}$ & -0.28 & 0.24 \\
\hline RBMT story $\%$ recall & 0.24 & 0.22 & -0.19 & -0.02 & $0.51 * *$ & $0.51 * *$ & -0.09 & -0.20 \\
\hline Visual Association Test & $0.40^{* *}$ & $0.40^{* *}$ & -0.22 & 0.15 & $0.55 * *$ & $0.53 * *$ & -0.14 & -0.15 \\
\hline Boston Naming Test & $0.36 * *$ & $0.38^{* *}$ & -0.14 & 0.13 & $0.29 *$ & $0.27^{+}$ & 0.16 & -0.12 \\
\hline Category fluency & $0.33 * *$ & $0.36^{* *}$ & -0.07 & 0.11 & 0.07 & 0.08 & -0.02 & -0.08 \\
\hline Letter fluency & $0.25^{*}$ & $0.29 * *$ & -0.11 & 0.05 & 0.15 & 0.11 & -0.03 & -0.23 \\
\hline mWCST concepts & -0.07 & -0.06 & 0.07 & 0.03 & 0.09 & 0.11 & 0.10 & -0.19 \\
\hline Trailmaking B-A & -0.01 & -0.05 & -0.07 & -0.07 & -0.17 & -0.17 & 0.11 & -0.17 \\
\hline Stroop III-II & -0.03 & -0.03 & 0.11 & -0.20 & $-0.09^{+}$ & -0.25 & 0.08 & $0.29^{+}$ \\
\hline Clock drawing & 0.10 & 0.13 & -0.05 & 0.14 & 0.16 & 0.10 & $-0.23^{+}$ & 0.19 \\
\hline
\end{tabular}

bvFTD, behavioral variant frontotemporal dementia; AD, Alzheimer's disease; con, control participants; A', discriminability; B", response bias. Data are Spearman correlation coefficients adjusted for age and level of education. ${ }^{*} p<.05, * * p<.01,{ }^{+} p<.1$.

a significantly lower discriminability on the recognition trial of the RAVLT than the control group. In turn, the AD group had a significantly lower discriminability than patients with bvFTD. Discriminative ability was mainly associated with memory for both patient groups and additionally associated with language but not executive functioning in bvFTD. Although both patients with bvFTD and AD also had a slightly more liberal response bias ("yes" tendency) than control participants, there was no difference in response bias between the two patient groups. Response bias was also unrelated to other cognitive measures in our analysis.

These results corroborate the growing body of evidence showing considerable episodic memory impairment in bvFTD (Hornberger et al., 2010). Multiple previous studies on delayed free recall and, to a lesser extent, immediate recall show that episodic memory can even be similarly impaired in bvFTD and AD (e.g., Gregory et al., 1997; Hornberger et al., 2010; Pennington et al., 2011; Ricci et al., 2012). Indeed, also in the present study, delayed recall performance was impaired in both bvFTD and AD. Much less is known about differences in recognition memory in differentiating AD from bvFTD, which is surprising considering the high prevalence of recognition memory paradigms in functional neuroimaging studies in dementia (Clement, Belleville, \& Mellah, 2010; Papma et al., 2017) and the fact that recognition memory is part of standard assessment of memory in clinical practice. Our results partly confirm findings from a recent study by Flanagan et al. (2016) showing a significantly increased false positive rate for both patients with $\mathrm{AD}$ and bvFTD compared to controls and no difference in the simple discrimination index ("hits minus false alarms") for patients with bvFTD versus $\mathrm{AD}$. In contrast, whereas Flanagan et al. showed that discriminative ability was associated with executive functioning (disinhibition), our results indicate an association with memory but not executive functioning (in both AD and bvFTD). This difference in involvement of executive functioning may be due to differences in the type of executive process that was measured (interference on the Stroop test $v s$. inhibition of a semantically constrained response in the Hayling test; Belleville, Rouleau, \& Van der Linden, 2006). In the patients with bvFTD, discriminability was associated with language performance, possibly resulting from the verbal nature of the RAVLT, and it also reflects the (sometimes striking) language deficits that are present in bvFTD (i.e., naming, word comprehension, and diminished propositional speech; Hardy et al., 2016).

Response bias B" was not previously examined in bvFTD, but Russo et al. (2017) report a significantly lower discriminative ability and a liberal response bias in patients with $\mathrm{AD}$, which is highly similar to our findings in the $\mathrm{AD}$ group. 
The lack of a difference in response bias between bvFTD and $\mathrm{AD}$ found in our study appears counterintuitive, but the (slightly) liberal response bias that was present in both patients groups (but not in controls) is in line with previous findings in patients with dementia (Beth et al., 2009). Possibly, the cause of the liberal response bias is different between $A D$ and bvFTD (overendorsement of a yes response in an uncertain situation $v s$. disinhibition/perseverative errors). In our view, the presence of an impaired discrimination index A' supports the presence of a "true" memory deficit in bvFTD, corroborating results from previous studies (Hornberger et al., 2012). Whereas patients with bvFTD outperformed patients with $\mathrm{AD}$ in delayed recall, for both $\mathrm{AD}$ and bvFTD the recognition score was significantly lower than the free recall score (relative to the control group), indicating the additional value of the recognition memory paradigm. Post hoc analysis in the groups of bvFTD patients showed no differences in discriminability or response bias between GRN and MAPT mutation carriers and C9orf72 repeat expansion carriers. This is surprising as increasing evidence indicates mutation-specific cognitive profiles in genetic bvFTD (e.g., Snowden et al., 2015) and may have resulted from the modest sample size in this subgroup analysis.

Strengths of the present study include the large and welldefined patient samples, the use of the RAVLT as widely used memory test, and involvement of both simple and elaborate measures of discriminative ability and response bias. Limitations include the lack of postmortem pathological confirmation in the patient groups, which is particularly problematic in bvFTD (Klatka, Schiffer, \& Powers, 1996; Varma et al., 1999), albeit that $40 \%$ of the patients with bvFTD had a known genetic mutation for bvFTD. Also, although analyses were adjusted for time since symptom onset, the disease course is different between AD and bvFTD which makes it difficult to truly match the patient groups (as is reflected in our study in lower age in bvFTD patients and lower MMSE scores in $\mathrm{AD}$ patients). Moreover, in our sample, time since symptom onset was shorter for bvFTD patients than for $\mathrm{AD}$, which reflects our role as an expertise center for FTD and inclusion of a proportion of mutation carriers from known FTD families in the Netherlands. One can hypothesize that differences in memory performance in bvFTD and $A D$ change with disease progression and accompanying atrophy of frontal and temporal brain areas. For example, it is hypothesized that frontal/dysexecutive impairment in $\mathrm{AD}$ increases as the disease progresses (Russo et al., 2017), which may result in a larger (liberal) response bias and a potential larger between-group difference. The mean age of the AD patients was relatively young (68.1 \pm 7.2 years) with an MMSE score of $22.7 \pm 4.6$ indicating a substantial number of early-onset cases and a relatively mild disease severity. Generalization of our results to older patients and more severe disease stages should therefore be performed with caution. We used the recognition trial of the RAVLT as it is one of the most commonly used verbal episodic memory tests that is easily administered and readily available in clinical practice. Our analyses were thus constrained by the methodological limitations of such a clinical tool, thereby also limiting generalizability of our results. The paradigm of the California Verbal Learning Test may be even better suited to examine the relative contribution of memory and executive processes in these patient groups as it allows for examination of (proactive and retroactive) interference and cueing. It would also be valuable to vary the conditions of the recognition memory paradigm (number and type of distractor items) and see how this influences discriminative ability and response bias in patients with dementia. An important clinical implication that results from our study is that although patients with $\mathrm{AD}$ show an overall worse recognition memory performance, the presence of a recognition memory deficit does not rule out bvFTD in individual patients.

In sum, our results show a difference in recognition memory performance between patients with bvFTD and $\mathrm{AD}$, particularly in discriminative ability, but not in response bias. Discriminative ability was mainly associated with memory for both patient groups and was additionally associated with language but not executive functioning in bvFTD. Response bias was unrelated to other cognitive functions.

\section{ACKNOWLEDGMENTS}

We would like to thank all participants and their families for taking part in our study. This work was supported by the Dioraphte Foundation (grant numbers 09-02-03-00); the Association for Frontotemporal Dementias Research Grant 2009; The Netherlands Organization for Scientific Research (NWO) grant HCMI (grant number 056-13-018); ZonMw Memorabel (Deltaplan Dementie project numbers 733051042 and 733050103); JPND PreFrontAls consortium project number 733051042; and Alzheimer Nederland and the Bluefield project. Several authors of this publication are members of the European Reference Network for Rare Neurological Diseases - Project ID No 739510.

\section{CONFLICT OF INTEREST}

The authors report no conflict of interest.

\section{SUPPLEMENTARY MATERIAL}

To view supplementary material for this article, please visit https://doi.org/10.1017/S1355617720000375

\section{REFERENCES}

Belleville, S., Rouleau, N., \& van der Linden, M. (2006). Use of the Hayling task to measure inhibition of prepotent response in normal aging and Alzheimer's disease. Brain and Cognition, 62, 113-119.

Bertoux, M., de Souza, L.C., Corlier, F., Lamari, F., Bottlaender, M., Dubois, B., \& Sarazin, M. (2014). Two distinct amnesic profiles in behavioral variant frontotemporal dementia. Biological Psychiatry, 75, 582-588. doi: 10.1016/j.biopsych.2013.08.017 
Beth, E.H., Budson, A.E., Waring, J.D., \& Ally, B.A. (2009). Response bias for picture recognition in patients with Alzheimer disease. Cognitive and Behavioral Neurology, 22, 229-235. doi: 10.1097/WNN.0b013e3181b7f3b1.

Budson, A.E., Wolk, D.A., Chong, H., \& Waring, J.D. (2006). Episodic memory in Alzheimer's disease: Separating response bias from discrimination. Neuropsychologia, 44, 2222-2232.

Clement, F., Belleville, S., \& Mellah, S. (2010). Functional neuroanatomy of the encoding and retrieval processes of verbal episodic memory in MCI. Cortex, 46, 1005-1015.

Corrigan, J.D. \& Hinkeldey, N.S. (1987). Relationships between parts A and B of the Trail Making Test. Journal of Clinical Psychology, 43, 402-409.

Dopper, E.G., Rombouts, S.A., Jiskoot, L.C., den Heijer, T., de Graaf, J.R., de Koning, I., \& van Swieten, J.C. (2014). Structural and functional brain connectivity in presymptomatic familial frontotemporal dementia. Neurology, 80:814-823.

Dubois, B., Slachevsky, A., Litvan, I., \& Pillon, B. (2000). The FAB: a frontal assessment battery at bedside. Neurology, 55, 1621-1626.

Flanagan, E.C., Wong, S., Dutt, A., Tu, S., Bertoux, M., Irish, M., \& Hornberger, M. (2016). False recognition in behavioral variant frontotemporal dementia and Alzheimer's disease-disinhibition or Amnesia? Frontiers in Aging Neuroscience, 8, 177. doi: 10. 3389/fnagi.2016.00177.

Folstein, M.F., Folstein, S.E., \& McHugh, P.R. (1975). "Minimental state". A practical method for grading the cognitive state of patients for the clinician. Journal of Psychiatric Research, 12, 189-198.

Frisch, S., Dukart, J., Vogt, B., Horstmann, A., Becker, G., Villringer, A., \& Schroeter, M.L. (2013). Dissociating memory networks in early Alzheimer's disease and Frontotemporal lobar degeneration - a combined study of hypometabolism and atrophy. PLoS One, 8:e55251. doi: 10.1371/journal.pone.0055251

Glosser, G., Gallo, J.L., Clark, C.M., \& Grossman, M. (2002). Memory encoding and retrieval in frontotemporal dementia and Alzheimer's disease. Neuropsychology, 16, 190-196.

Gregory, C.A., Orrell, M., Sahakian, B., \& Hodges, J.R. (1997). Can frontotemporal dementia and Alzheimer's disease be differentiated using a brief battery of tests?. International Journal of Geriatric Psychiatry, 12, 375-383.

Hardy, C.J., Buckley, A.H., Downey, L.E., Lehmann, M., Zimmerer, V.C., Varley, R.A., \& Warren, J.D. (2016). The language profile of behavioral variant frontotemporal dementia. Journal of Alzheimers Disease, 50, 359-371. doi: 10.3233/ JAD-150806.

Heidler-Gary, J. \& Hillis, A.E. (2007). Distinctions between the dementia in amyotrophic lateral sclerosis with frontotemporal dementia and the dementia of Alzheimer's disease. Amyotrophic Lateral Sclerose, 8, 276-282.

Hornberger, M. \& Piguet, O. (2012). Episodic memory in frontotemporal dementia: a critical review. Brain, 135, 678-692. doi: 10.1093/brain/aws011.

Hornberger, M., Piguet, O., Graham, A.J., Nestor, P.J., \& Hodges, J.R. (2010). How preserved is episodic memory in behavioral variant frontotemporal dementia? Neurology, 74, 472-479.

Irish, M., Piguet, O., Hodges, J.R., \& Hornberger, M. (2014). Common and unique gray matter correlates of episodic memory dysfunction in frontotemporal dementia and Alzheimer's disease. Human Brain Mapping, 35, 1422-1435. doi:10.1002/hbm.22263 Kaplan, E., Goodglass, H., \& Weintraub, S. (1978). The Boston Naming Test. Philadelphia: Lea \& Febiger.
Klatka, L.A., Schiffer, R.B., \& Powers, J.M. (1996). Incorrect diagnosis of Alzheimer's disease: A clinicopathological study. Archives of Neurology, 53, 35-42.

Kramer, J.H., Rosen, H.J., Du, A.T., Schuff, N., Hollnagel, C., Weiner, M.W., \& Delis, D.C. (2005). Dissociations in hippocampal and frontal contributions to episodic memory performance. Neuropsychology, 19, 799-805.

Lemos, R., Duro, D., Simões, M.R., \& Santana, I. (2014). The free and cued selective reminding test distinguishes frontotemporal dementia from Alzheimer's disease. Archives of Clinical Neuropsychology, 29, 670-679. doi: 10.1093/arclin/acu031.

Lindeboom, J., Schmand, B., Tulner, L., Walstra, G., \& Jonker, C. (2002). Visual association test to detect early dementia Alzheimer type. Journal of Neurology, Neurosurgery, and Psychiatry, 73, 126-133.

McKhann, G.M., Knopman, D.S., Chertkow, H., Hyman, B.T., Jack, C.R. Jr, Kawas, C.H., \& Phelps, J.J. (2011). The diagnosis of dementia due to Alzheimer's disease: recommendations from the National Institute on Aging-Alzheimer's Association workgroups on diagnostic guidelines for Alzheimer's disease. Alzheimer's and Dementia, 7:263-9.

Neary, D., Snowden, J.S., Gustafson, L., Passant, U., Stuss, D., Black, S., \& Benson, D.F. (1998). Frontotemporal lobar degeneration: a consensus on clinical diagnostic criteria. Neurology, 51, 1546-1554.

Nelson, H.E. (1976). A modified card sorting test sensitive to frontal lobe defects. Cortex, 12, 313-324.

Ossenkoppele, R., Pijnenburg, Y.A., Perry, D.C., Cohn-Sheehy, B.I., Scheltens, N.M., Vogel, J.W., \& Rabinovici, G.D. (2015). The behavioural/dysexecutive variant of Alzheimer's disease: clinical, neuroimaging and pathological features. Brain, 138, 2732-49.

Papma, J.M., de Groot, M., de Koning, I., Mattace-Raso, F.U., van der Lugt, A., Vernooij, M.W., \& Smits, M. (2014). Cerebral small vessel disease affects white matter microstructure in mild cognitive impairment. Human Brain Mapping, 35, 2836-51. doi: 10.1002/hbm.22370.

Papma, J.M., Smits, M., de Groot, M., Mattace Raso, F.U., van der Lugt, A., Vrooman, H.A., \& Prins, N.D. (2017). The effect of hippocampal function, volume and connectivity on posterior cingulate cortex functioning during episodic memory fMRI in mild cognitive impairment. European Radiology, 27, 3716-3724.

Pasquier, F., Grymonprez, L., Lebert, F., \& Van der Linden, M. (2001). Memory impairment differs in frontotemporal dementia and Alzheimer's disease. Neurocase, 7, 161-71.

Pennington, C., Hodges, J.R., \& Hornberger, M. (2011). Neural correlates of episodic memory in behavioral variant frontotemporal dementia. Journal of Alzheimer's Disease, 24, 261-268. doi: 10.3233/JAD-2011-101668.

Poos, J.M., Jiskoot, L.C., Papma, J.M., van Swieten, J.C., \& van den Berg, E. (2018). Meta-analytic review of memory impairment in behavioral variant frontotemporal dementia. Journal of the International Neuropsychological Society, 24, 593-605.

Rascovsky, K., Hodges, J.R., Knopman, D., Mendez, M.F., Kramer, J.H., Neuhaus, J., Van Swieten, J.C., Seelaar, H., Dopper, E.G.P., Onyike, C.U., Hillis-Trupe, A., Josephs, K.A., Boeve, B.F., Kertesz, A., Seeley, W.W., Rankin, K.P, Johnson, J.K., Gorno-Tempini, M.L., Rosen, H., Prioleau-Latham, C.E., Lee, A., Kipps, C.M., Lillo, P., Piguet, O., Rohrer, J.D., Rossor, M.N., Warren, J.D., Fox, N.C., Galasko, D., Salmon, D.P., Black, S.E., Mesulam, M., Weintraub, S., Dickerson, B.C., 
Diehl-Schmid, J., Pasquier, F., Deramecourt, V., Lebert, F., Pijnenburg, Y., Chow, T.W., Manes, F., Grafman, J., Cappa, S.F., Freedman, M., Grossman, M., \& Miller, B.L. (2011). Sensitivity of revised diagnostic criteria for the behavioural variant of frontotemporal dementia. Brain, 134, 2456-2477.

Ricci, M., Graef, S., Blundo, C., \& Miller, L.A. (2012). Using the Rey Auditory Verbal Learning Test (RAVLT) to differentiate Alzheimer's dementia and behavioural variant fronto-temporal dementia. The Clinical Neuropsychologist, 26, 926-941. doi: 10.1080/13854046.2012.704073.

Rohrer, J.D. \& Rosen, H.J. (2013). Neuroimaging in frontotemporal dementia. International Review of Psychiatry, 25,221-229.

Royall, D.R., Cordes, J.A., \& Polk, M. (1998). CLOX: an executive clock drawing task. Journal of Neurology Neurosurgery and Psychiatry, 64, 588-594.

Rugg, M.D., Otten, L.J., \& Henson, R.N. (2002). The neural basis of episodic memory: evidence from functional neuroimaging. Philosophical Transaction of the Royal Society B: Biological Sciences, 357, 1097-1110.

Russo, M.J., Cohen, G., Campos, J., Martin, M.E., Clarens, M.F., Sabe, L., \& Allegri, R.F. (2017). Usefulness of discriminability and response bias indices for the evaluation of recognition memory in mild cognitive impairment and Alzheimer disease. Dementia and Geriatric Cognitive Disorders, 43, 1-14. doi: 10.1159/000452255.

Schmand, B., Groenink, S.C., \& van den Dungen, M. (2008). Letter fluency: psychometric properties and Dutch norms (in Dutch). Gerontologie en Geriatrie, 39, 64-74.

Snodgrass, J.G. \& Corwin, J. (1988). Pragmatics of measuring recognition memory: applications to dementia and amnesia. Journal of Experimental Psychology: General, 117, 34-50.

Snowden, J.S., Adams, J., Harris, J., Thompson, J.C., Rollinson, S., Richardson, A., \& Pickering-Brown, S. (2015). Distinct clinical and pathological phenotypes in frontotemporal dementia associated with MAPT, PGRN and C9orf72 mutations. Amyotrophic Lateral Sclerosis and Frontotemporal Degeneration, 16, 497-505.
Steketee, R.M., Meijboom, R., de Groot, M., Bron, E.E., Niessen, W.J., van der Lugt, A., \& Smits, M. (2016). Concurrent white and gray matter degeneration of disease-specific networks in early-stage Alzheimer's disease and behavioral variant frontotemporal dementia. Neurobiology of Aging, 43, 119-128.

Stroop, J.E. (1935). Studies of interference in serial verbal reactions. Journal of Experimental Psychology, 18, 643-662.

Thompson, J.C., Stopford, C.L., Snowden, J.S., \& Neary, D. (2005). Qualitative neuropsychological performance characteristics in frontotemporal dementia and Alzheimer's disease. Journal of Neurology, Neurosurgery, and Psychiatry, 76, 920-927.

van der Elst, W., van Boxtel, M.P., van Breukelen, G.J., \& Jolles, J. (2005). Rey's Verbal Learning Test: normative data for 1,855 healthy participants aged 24-81 years and the influence of age, sex, education, and mode of presentation. The Journal of the International Neuropsychological Society, 11, 290-302.

Varma, A.R., Snowden, J.S., Lloyd, J.J., Talbot, P.R., Mann, D.M., \& Neary, D. (1999). Evaluation of the NINCDSADRDA criteria in the differentiation of Alzheimer's disease and frontotemporal dementia. Journal of Neurology, Neurosurgery, and Psychiatry, 66, 184-188.

Verhage, F. (1964) Intelligence and Age: Study with Dutch People Aged 12-77 (in Dutch). Assen: Van Gorcum.

Wilson, B.A., Cockburn, J., \& Baddeley, A. (1985). Rivermead Behavioural Memory Test. London: Thames Valley Test Company.

Wong, S., Flanagan, E., Savage, G., Hodges, J.R., \& Hornberger, M. (2014). Contrasting prefrontal cortex contributions to episodic memory dysfunction in behavioural variant frontotemporal dementia and Alzheimer'sdisease. PLOS ONE, 9, e87778.

Zakzanis, K.K. (2001). Statistics to tell the truth, the whole truth, and nothing but the truth: Formulae, illustrative numerical examples, and heuristic interpretation of effect size analyses for neuropsychological researchers. Archives of Clinical Neuropsychology, $16,653-667$. 